\title{
The Missing Satellite Problem Outside of the Local Group
}

\section{Masayuki Tanaka ${ }^{1}$, Masashi Chiba, Yutaka Komiyama, Mikito Tanaka, Sakurako Okamoto and Takashi Okamoto}

\author{
${ }^{1}$ National Astronomical Observatory of Japan, 2-21-1 Osawa, Mitaka, Tokyo 181-8588, Japan \\ email: masayuki.tanaka@nao.ac.jp
}

\begin{abstract}
We report on the first results from our pilot observation of nearby galaxies with Hyper Suprime-Cam. We have observed two galaxies with mass similar to that of the Milky Way Galaxy and measured the abundance of their satellite galaxies in order to address the missing satellite problem outside of the Local Group. We find that (1) the abundance of dwarf galaxies is smaller by a factor of two than the prediction from one of the current hydro-dynamical simulations and (2) there is a large halo to halo scatter. Our results highlight the importance of a statistical sample of nearby galaxies to address the missing satellite problem.
\end{abstract}

Keywords. galaxies:dwarf, cosmology:observations

\section{Introduction}

The $\Lambda$ CDM model is widely accepted as a standard cosmological model. It has passed many stringent observational tests and it reproduces the large-scale matter distribution in the Universe that we see today extremely well. However, the $\Lambda$ CDM model is not without problems - it has a few possible flaws on small scales such as the cusp-core problem, toobig-to-fail problem (Boylan-Kolchin et al. 2011), and missing satellite problem (Moore et al. 1999; Klypin et al. 1999). We do not have satisfactory solutions to these problems and they may motivate us to adopt other models such as WDM and self-interacting DM.

This paper aims at addressing the missing satellite problem - the more than an order of magnitude shortage of observed dwarf galaxies around the Mikly Way (MW) and M31 compared to theoretical expectations - with an unprecedented statistical accuracy. The problem was first pointed out in 1999 based on (DM-only) N-body simulations. Since then, there has been tremendous progress in hydro-dynamical simulations of galaxies in a cosmological context and recent simulations show that, once baryonic effects such as star formation and SNe feedback are incorporated, not all the satellite halos host dwarf galaxies and the tension between the observed and expected numbers of dwarf galaxies is significantly reduced (e.g., Sawala et al. 2016). Although the baryonic effects may be one of the solutions to the missing satellite problem, we should point out that the problem has been often addressed in the Local Group (LG hereafter), assuming that the MW and M31 are typical halos in the Universe. This is a big assumption because there is a large diversity in the accretion history of halos - some halos assemble most of their mass at early times, while others assemble late. Due to this statistical nature of halos, any cosmological tests must be performed with statistical rigor.

We have initiated a project to observationally test the missing satellite problem beyond the LG and this paper presents our first results from the program. 


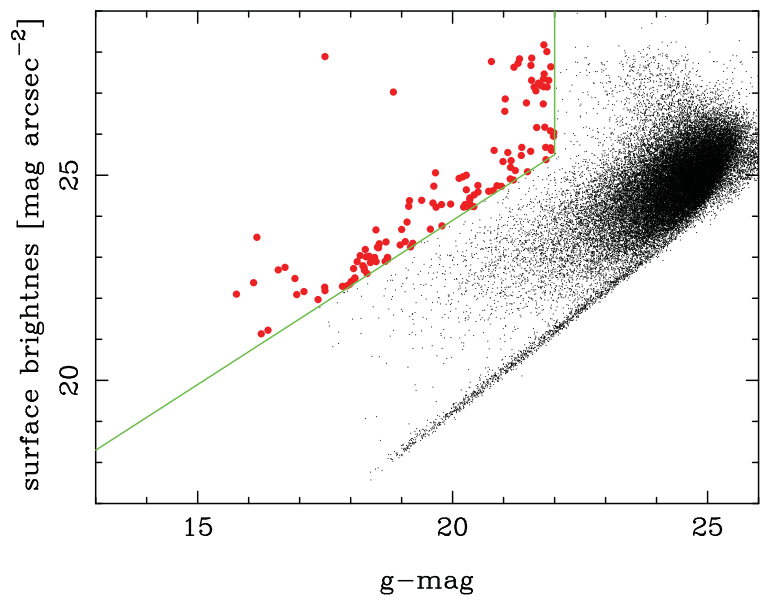

Figure 1. Surface brightness plotted against magnitude in the $g$-band for a field around one of the host galaxies. Objects in the top-left corner are the dwarf galaxy candidates and this cut is carefully chosen to include the LG dwarf galaxies when observed at $\sim 20 \mathrm{Mpc}$.

\section{Observation and Dwarf Galaxy Selection}

As a pilot observation, we observed several nearby MW-like galaxies with the Hyper Suprime-Cam (HSC). The target galaxies were carefully selected to satisfy the following criteria: (a) the distance is known with a reasonable accuracy and is located at $\lesssim 20 \mathrm{Mpc}$, (b) the virial radius $\left(r_{200}\right)$ can be covered within a single HSC pointing, (c) there are no very bright $\left(m_{g} \lesssim 6\right)$ stars within the field of view, and (d) the Galactic extinction is relatively small $\left(a_{r} \lesssim 0.1 \mathrm{mag}\right)$. We observed the galaxies in $g$ and $i$-bands for $\sim 25$ minutes each.

Due to the close distances to the targets, dwarf galaxies can be selected as low-surface brightness galaxies (Fig. 1). We use the same selection for all the galaxies here because they are located at similar distances. Background face-on spiral galaxies as well as fake sources also pass the selection, but they can easily be eliminated by eye, provided that the seeing is good - the image quality of our data is 0.6 arcsec. Poor seeing data would not allow us to cleanly eliminate the background contamination. We use blank field data as a control field sample and field dwarf galaxies (i.e., those do not belong to any massive galaxies) are statistically subtracted from the target sample. In Fig. 2, we show a few random examples of the selected dwarf galaxies.

\section{Results and Discussion}

The resultant luminosity functions (LFs) of the dwarf galaxies are shown in Fig. 3 . Here we show LFs of two nearby galaxies: NGC2950 and NGC779. They are located at similar distances $(\sim 21 \mathrm{Mpc})$ and also have similar halo mass $\left(\sim 2 \times 10^{12} \mathrm{M}_{\odot}\right.$, which is inferred from the stellar mass of these galaxies using the parameterization by Moster et al. (2010). Note that the object detection incompleteness and areas masked due to bright stars are corrected for in these LFs. For comparison, we also show LF of MW McConnachie (2012) and those from cosmological SPH simulations by Okamoto (2013).

Fig. 3 suggests that (1) the observed LFs are smaller on average by a factor of 2 compared to the simulation albeit with very small number statistics, and (2) both simulation and observation hint at a large diversity in LFs in halos of similar mass. The observed diversity is not surprising because each halo has different formation and accretion 

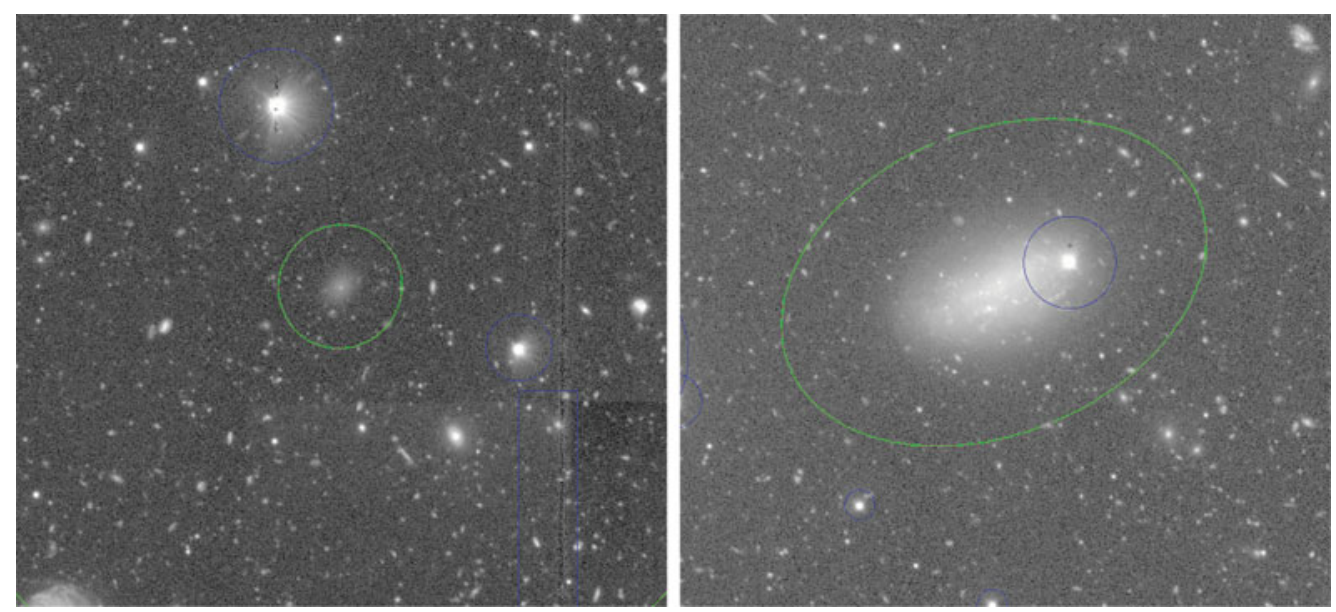

Figure 2. Sample images of the dwarf galaxies that we selected. The circles at the center indicate the selected dwarfs and those around bright stars are masked areas.

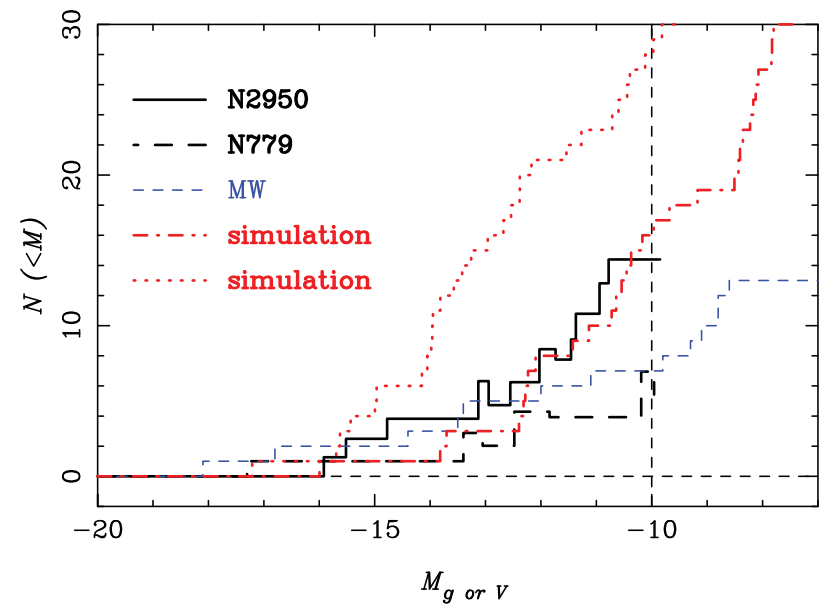

Figure 3. Cumulative LFs of the observed dwarf galaxies (solid and dashed lines), MW (thin dashed), and those from simulations (dotted and dot-dashed; these two halos are drawn from the Aquarius simulation). The observed LFs go down at a few places due to the statistical field subtraction. All the central galaxies considered here have similar halo mass of $\sim 10^{12} \mathrm{M}_{\odot}$. Note the large diversity of the LFs both in observation and simulation.

histories. It is this diversity that motivates us to go beyond the LG to statistically address the missing satellite problem. We are now collecting more data to construct a large statistical sample of nearby galaxies.

\section{References}

Boylan-Kolchin, M., Bullock, J. S., \& Kaplinghat, M. 2011, MNRAS, 415, L40

Klypin, A., Kravtsov, A. V., Valenzuela, O., \& Prada, F. 1999, ApJ, 522, 82

McConnachie, A. W. 2012, AJ, 144, 4

Moore, B., Ghigna, S., Governato, F., et al. 1999, ApJ (Letters), 524, L19

Moster, B. P., Somerville, R. S., Maulbetsch, C., et al. 2010, ApJ, 710, 903

Okamoto, T. 2013, MNRAS, 428, 718

Sawala, T., Frenk, C. S., Fattahi, A., et al. 2016, MNRAS, 456, 85 